\title{
Semiquantitative Analysis of Ischemic Dysfunctional Myocardial Tissue: Comparison of Contrast Enhanced MRI and Myocardial PET
}

\author{
Federica Groppo Marchisio ${ }^{1}$, Stephane Chauvie ${ }^{2}$, Maurizio Grosso ${ }^{1}$, Alberto Biggi², \\ Bobbio Marco ${ }^{3}$ and Mauro Feola ${ }^{*}, 4$
}

\author{
${ }^{I}$ Radiology Department, ${ }^{2}$ Nuclear Medicine Service and ${ }^{3}$ Cardiology Division Ospedale S. Croce-Carle Cuneo and \\ ${ }^{4}$ Cardiovascular Rehabilitation-Heart Failure Unit, Ospedale SS Trinita', Fossano, Italy
}

\begin{abstract}
Cardiac Positron Emission Tomography (PET) has been demonstrated to be highly specific in differentiating viable from scarred myocardium in patients with ischemic left ventricular dysfunction. The aim of this study was to compare PET imaging with contrast-enhanced magnetic resonance imaging (ceMRI) in patients with ischemic myocardial dysfunction, analysing semiquantitatively the amount of non-viable myocardial tissue.

Results: 10 patients with left ventricle ischemic dysfunction underwent myocardial PET with ${ }^{13} \mathrm{~N}$-ammonia/FDG and delayed sequences of cardiac MRI after injection of gadolinium diethyltriaminepentaacetic acid (Gd-DTPA). PET and MRI images were divided into 17 segments and examined by 2 independent observers. Extent of tissue damage was estimated using a 5point scoring system. Using PET as gold standard, accuracy in detecting scar tissue was determined for ceMRI. High agreement rate $(63 \%$ and $69 \%)$ between myocardial necrosis in PET and ceMRI was evaluated by Kappa test. Comparison between scores in transmutable extension in PET/ceMRI study with a " 0 tolerance" system (that is considering results agreement only if overlap results), the 2 blinded observers demonstrated an agreement rate of $68.9 \%$ and $76.5 \%$ respectively, with a difference of 7.6\% [IC 95\% from - 2 to 17.1]. With one point of tolerance (considering results agreement even if there was one point of difference), an agreement rate of 93.6 and $95.3 \%$ emerged, with a difference of $1.8 \%$ [IC $95 \%$ from -3.1 to $6.6 \%]$.

Conclusions: In the identification of non-viable myocardium, semiquantative analysis of cardiac PET and ceMRI obtained similar accuracy of the two imaging techniques.
\end{abstract}

Keywords: Contrast enhanced MRI, myocardial dysfunction, myocardial positron emission tomography.

\section{INTRODUCTION}

Acute myocardial infarction, which occurs when there is a prolonged occlusion of an epicardial coronary artery without sufficient collateral blood supply, is the most common cause of death and one of the most common causes of morbidity in industrialized nations [1]. For survivors of myocardial infarction, the accurate assessment of damage, as well as the identification of the residual tissue viability in patients with acute myocardial infarction and chronic ischemic heart disease, is crucial in planning future treatment and individual risk stratification [2]. In fact, identification, differentiation and quantification of reversible and irreversible myocardial damage in patients with reduced left ventricular ejection fraction (LVEF) and coronary artery disease are useful in order to predict which patients would obtain profit from revascularization, such as increase of ejection fraction and rate of survival since patients with dysfunctional but viable myocardium profit from revascularization $[3,4]$ and affects both short-term and long term prognosis [5-8]. Several techniques analyzing the morphologic [9], functional [10], cellular and metabolic [11] integrity of the myocardium have been introduced into clinical medicine. Each of these

\footnotetext{
*Address correspondence to this author at the Cardiovascular RehabilitationHeart Failure Unit, Ospedale SS Trinita', Via Ospedale 4, 12045 Fossano (CN), Italy; E-mail: m_feola@virgilio.it
}

methods have specific advantages for the diagnosis of myocardial viability, but none allows direct visualization of the transmural extent of viable and non-viable tissue. Detection of viability based on assessment of myocardial perfusion and glucose metabolism by PET is now considered the reference method [11], and many studies suggest that MRI using gadolinium-based contrast agent (ceMRI) can delineate reversible and irreversible damaged myocardium and can predict areas which will recover functionally after revascularization [12-14] as well as metabolic study by PET. ceMRI of the heart is a non-invasive diagnostic modality with high spatial resolution, an excellent safety profile of the contrast agent, and a lack of radiation exposure, which has recently been proposed as an alternative imaging modality for the assessment of myocardial viability [15]. Among available imaging techniques, ceMRI is one of the favourite applications of both imagers and referring physicians. The so called "late enhancement" concept, also referred to as "delayed enhancement" or "late gadolinium enhancement", has obtained with a T1-weighted imaging of the myocardium in the late equilibrium phase after intravenous administration of gadolinium-based contrast medium. Inversion recovery (IR) sequences have been proved reliable and robust in this respect. Several histological studies have determined a relationship between hyperenhanced myocardium at ceMRI and necrosis $[12,16,17]$, and ceMRI permits to distinguish between regions with reversible and irreversible myocardial injury, 
depending on the transmural extent of hyperenhancement, which has been shown to be predictive of recovery of contractile function after myocardial revascularization [18, 19]. The aim of this study was to compare extent and localization of hyperenhancement in Delayed Enhancement (DE) ceMRI with nonviable tissue defined by nuclear metabolic imaging using ${ }^{18} \mathrm{~F}$-fluorodeoxyglucose (FDG) positron emission tomography (PET) in patients with ischemic heart disease and left ventricular dysfunction.

\section{METHODS}

\section{Patients}

From March 2006 to September 2007, patients with coronary artery disease (CAD) proved by coronary angiography and left ventricular systolic dysfunction $(\mathrm{LVEF}<40 \%)$ were included in the study. Myocardial PET and MRI were performed in the same subject within 1 week, without any significant change in clinical status.

\section{ceMRI}

The protocol consisted of contrast-enhanced MRI to determine the extent of delayed contrast enhancement.

Cardiac MRI was performed with a clinical 1.5-T Gyroscan ACS-NT MRI scanner, Philips Medical System, Eindhoven, The Netherlands. A four-element cardiac phasedarray receiver surface coil was utilized for signal reception. A breath-hold segmented fast gradient-echo imaging employing steady-state acquisition (Balanced Echo), electrocardiographically triggered sequence was performed to evaluate global left ventricular function using standard parameters. In each patient, a total of nine to 12 short-axis views (depending on left ventricular volumes) and two longaxis views (vertical and horizontal) were acquired with a minimum of 30 cine frames/slices. Delayed sequences were obtained approximately 12 minutes after intravenous injection of $0.2 \mathrm{mmol} / \mathrm{kg}$ gadolinium diethyltriaminepentaacetic acid (Gd-DTPA) using a fast field echo sequence (slice thickness $8 \mathrm{~mm}$, FOV $360 \mathrm{~mm}$, flip angle $15^{\circ}$, TE $1.3 \mathrm{~ms}$, TR) $4.1 \mathrm{~ms}$. Images were acquired at end-diastole in the same views. Delayed enhancement images were displayed with a grey scale to optimally show normal myocardium and the region of delayed-enhancement myocardium. The scan was analysed according to: a) the left ventricular function (sequences balanced-echo cine MRI) and b) the presence of scar tissue with delayed-enhancement (DE) images.

The extent of hyperenhancement was divided from transmural to subendocardial into 5 steps of a visual scoring system (0-4):

$0=\quad$ no delayed enhancement

$1=1-25 \%$ of myocardial wall with hyperenhancement

$2=\quad 26-50 \%$ of myocardial wall with hyperenhancement

$3=51-75 \%$ of myocardial wall with hyperenhancement

$4=\quad 76-100 \%$ of myocardial wall with hyperenhancement

A myocardial segment end-diastolic wall thickness $>6 \mathrm{~mm}$ (obtained with a fast gradient-echo sequence) with a visual score $>2$ was classified as necrotic segment.

\section{PET Imaging}

After an overnight fast, patients were injected with 585 $\mathrm{MBq}$ of ${ }^{13} \mathrm{~N}$-ammonia for the determination of myocardial perfusion at rest. A second PET study was performed in the same day, one hour after the injection of $378 \mathrm{MBq}$ FDG for cell metabolism while the patients were on a euglycemic hyperinsulinemic clamp (see below). PET images were obtained with a GE Discovery LS hybrid PET/CT system (GE Healthcare, Waukesha, Wisconsin). A CT scan was obtained for attenuation correction designed to reduce artefacts of cardiac motion using a helical scan with a rotation time of 1 second and an $11.25 \mathrm{~mm} /$ rotation bed speed. In such a way the $\mathrm{CT}$ acquisition of the heart was obtained in 27.5 seconds. All the PET studies were performed in 2D mode for scatter reduction. All images were reconstructed with an OSEM 28subset, 3-iteration method with an in-plane image resolution of $4.8 \mathrm{~mm}$ full width at half maximum (FWHM). The reconstruction matrix was $128 \times 128$ pixels with a $3.9 \mathrm{~mm}$ pixel size.

The FDG/NH $/ \mathrm{NH}_{3}$ images were evaluated semiquantitatively using a five-point scoring system dividing the left ventricle into 17 segments according to the recommendations of the American Heart Association [8]:

$\begin{array}{ll}0= & \text { normal tracer uptake (from } 90 \text { to } 100 \%) \\ 1= & \text { important (non transmural) tracer uptake } \\ 2= & \text { mild tracer uptake } \\ 3= & \text { severe lack of tracer uptake } \\ 4= & \text { no tracer uptake }\end{array}$

Viable myocardium was identified in myocardial segments with end-diastolic wall thickness $>6 \mathrm{~mm}$ exhibiting normal or near normal ${ }^{18} \mathrm{~F}-\mathrm{FDG}$ accumulation (PET uptake scores ranging from $0-2)$ with reduced $(<2.5)$ standard deviations (SD) with respect to normal ${ }^{13} \mathrm{~N}$-ammonia myocardial blood flow (mismatched pattern of imaging). Non-viable myocardium was verified in segments with an end-diastolic wall thickening $<6 \mathrm{~mm}$ or with severe/absent ${ }^{18} \mathrm{~F}$-FDG myocardial uptake scores of 3 to 4 with a reduced ${ }^{13} \mathrm{~N}$ ammonia myocardial blood flow (matched defect).

For each patient, the 17 segments were scored and the sum was calculated.

\section{Images Analysis}

The left ventricle was divided into 17 segments using the apex and a representative apical, equatorial and basal short and long-axis view, according to the standards for plane selection proposed by the American Heart Association, the American College of cardiology and Society of Nuclear Medicine [20] (Fig. 1). Images were assessed in both modalities within each segment by 2 independent and blinded observes.

\section{STATISTICAL ANALYSIS}

Images were assessed with the 5-point scoring system for both modalities within each segment by 2 independent observes, blinded to the results of the other observer and the other modality.

Using PET as the gold standard, accuracy in detecting scar tissue was determined for MRI. Inter-modalities agreement was made with both "0 tolerance" system and "1 tolerance" 


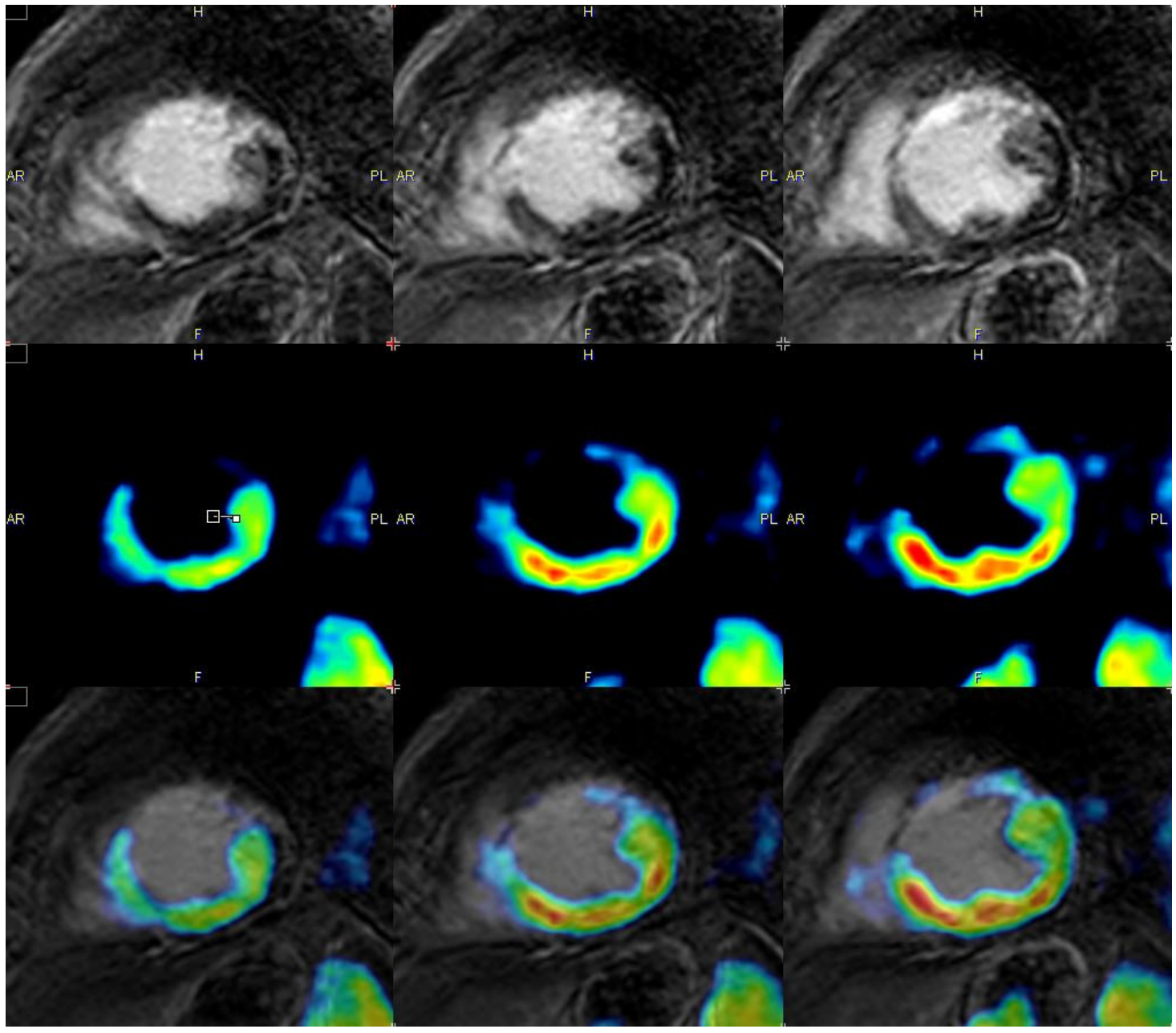

Fig. (1). FDG PET and ceMRI images of one patients with a previous anterior myocardial infarction. The upper images were obtained with ceMRI and represent a short axis of the mid left ventricle. In this image the presence of a transmural delayed hyperenhancement after gadolinium injection in mid ventricular anteroseptal and anterior segments emerged; in the mid lateral segment the delayed hyperehenancement was evidenced in the subendocardial tissue. In the middle, an FDG PET scan demonstrated an absence of uptake of the tracer in mid ventricular anteroseptal and anterior segment, with a clear reduction of uptake in mid lateral segment. In the lowest image the two images were summed, evidencing the correspondence of the non-viable area.

system, which means that results agreement was established only if results overlap ("tolerance 0 " system), or even if there was one point difference between results ("tolerance 1" system), which is considered still clinically acceptable.

Using PET scoring as the gold standard, the 2 observers have given MR images scores using a similar 5-point system, in order to analyze agreement between the 2 studies results, and establish if myocardial territories with no metabolism left in PET, so considered necrotic tissue, were showing scar tissue in MR study. Agreement between myocardial necrosis in PET findings and scar tissue observed in MRI has been evaluated by Kappa test [21]. After analyzing intermodalities agreement, and after comparing the 2 observers results given to globally considered myocardium, scores comparison was performed considering single myocardial territories perfused by different arteries: left anterior descending artery (LAD), right coronary artery (RCA) and left circumflex artery (LCX). This part of the study was performed by 2 observers and using a "tolerance 0 " and "tolerance 1" scoring system as well.
After that, still using Kappa test, agreement rate of both observers in giving negative (normal) and positive (pathological) scores in PET and MR studies, considering PET findings as gold standard, was evaluated.

$\mathrm{K}=(\mathrm{P} 0-\mathrm{Pc}) /(1-\mathrm{Pc})$

where

$\mathrm{K}=$ agreement index

P0 $=$ agreement rate observed between true positive and true negative, that is between positive and negative scores both in PET findings and MRI study. $\mathrm{Pc}=$ agreement rate expected between true positive and true negative, that is between positive and negative scores both in PET findings and MRI study.

\section{RESULTS}

Ten patients $(9$ men, from 46 to 77 years old, mean age $65 \pm 5$ ) were included in the study after giving informed consent. All the patients had documented a previous 
(>6-month) history myocardial infarction (7 anterior, 1 apical, 1 inferior and 1 non-q). All the selected patients had risk factors for ischemic heart disease (past history of nicotine abuse, diabetes mellitus, arterial hypertension, hypercholesterolemia, family history of sudden cardiac death). Coronary angiography assessed that 3 patients had 3 -vessel, 5 patients 2 vessel, and 2 patients had 1-vessel disease.

The semiquantitative analysis demonstrated that $72 / 170$ (42.3\%) left ventricular segments showed a significant reduction in FDG uptake (score $\geq 2$ ) whereas in 69/170 (40.6\%) segments a delayed hyperenhancement at ceMRI emerged. Comparison between scores in transmural extension of ${ }^{18} \mathrm{FDG}$ defect and hyperenhancement in ceMRI study, independently of territories of coronary perfusion, with a " 0 tolerance" system (that is considering results agreement only if overlap results) demonstrated an agreement rate of al $68.9 \% \mathrm{e}$ $76.5 \%$ respectively, with a difference of $7.6 \%$ [IC $95 \%$ from 2 to 17.1]. With one point of tolerance (considering results agreement even if there was one point of difference, "1 tolerance" system), the 2 observers had an agreement rate of 93.6 and $95.3 \%$, with a difference of $1.8 \%$ [IC $95 \%$ from -3.1 to $6.6 \%$ ] emerging from the comparison between PET and MRI scores.

Therefore the semiquantitative analysis obtained by two blinded observers gained similar results in giving scores of transmural necrosis in PET and MRI studies, underlining that myocardial necrosis observed in PET was confirmed in MRI study, with an overlapping of PET and MRI results with a close relationship between transmural uptake of contrast medium in MRI and uptake of 18FDG in PET. There was no significant interobservers variability for MRI and PET studies. About the second part of the study, that is the comparison of scores in different myocardial territories divided into different coronary distribution, analyzing myocardial territories served by left anterior descending artery (LAD), without inserting one point of tolerance ("0 tolerance" system), we have observed that the 2 observers had a concordance between PET and MRI scores of $68.6 \%$ and $78.6 \%$ respectively, with an interobservers difference rate of $10 \%$ [IC $95 \%$ from -0.4 to $24 \%]$. With a "1 tolerance" system, agreement between PET and MRI of the 2 observers was $97.1 \%$, with no difference [IC $95 \%$ from -5.5 to 5.5$]$.

Agreement analyzing myocardial territories perfused by left circumflex artery (LCX) was 70 and $78 \%$, with an interobservers difference of $8 \%$ [IC $95 \%$ (from -9 to $25 \%$ )] with a " 0 tolerance 2 system, and 90 and $92 \%$ respectively with a "tolerance 1" system, with a difference of $2 \%$ [IC 95\% from -9.2 to 13.2].

Results of myocardial territories perfused by right coronary artery (RCA), confirming previous studies trend, have given an agreement rate of PET and MRI scores of 68 and $72 \%$, with a difference of $29.4 \%$ [IC $95 \%$ from - 14 to 22] with a " 0 tolerance" system, and a rate of 98 and $96 \%$, with a difference of $4 \%$ [IC 95\% from -5.3 to 13.3 ] inserting one point of variability.

Using PET as the gold standard, the agreement rate between scores given by the 2 observers in positive (pathological) and negative about intramyocardial extension of necrosis in PET and MRI studies has been calculated. We have considered true positive scores established positive in
PET and confirmed in ceMRI, true negative scores negative both in PET and MRI, false positive scores positive in MRI but not in PET and false negative scores false in MRI but positive in PET. Comparing rates of agreement observed and expected between the 2 modalities of study by Kappa test, an agreement rate of $63 \%$ and $69 \%$ emerged. This result has to be considered satisfactorily by Karran system [21], considering very low an agreement rate lower than $20 \%$, low a rate from 20 to $40 \%$, mild a rate from 40 and $60 \%$, elevated from 60 to $80 \%$, and very high when higher than $80 \%$.

\section{DISCUSSION}

An accurate evaluation of viability and the amount of damage in patients surviving myocardial infarction in patients with ischemic left ventricular dysfunction is crucial for the therapeutic decision and for the stratification of the individual risk [22]. Identification, differentiation and quantification of myocardial damage in reversing and irreversible way are useful to predict which patient is potentially able to get benefits by revascularization, improving LVEF and survival $[6-8,23]$. In fact, despite the perioperative risk is increased in such patients, the surgical revascularization seemed to improve the left ventricular function and the prognosis. For the identification of the amount of residual viable tissue after an ischemic event numerous techniques of morphological, functional and cellular analysis and of the metabolic integrity of myocardium have been proposed [11, 24]. The noninvasive methods for detecting the presence of myocardial viability include myocardial PET (using NH3 for blood flow and ${ }^{18} \mathrm{FDG}$ for metabolism) and the stress echocardiography during dobutamine infusion. Recently, ceMRI has been proposed for the quality of images with good correlation with extension, location and the form of the ischemic lesion observed in histological examination. Previous experiences [14-16] showed as the area of myocardial lesion improved the intensity of signal up to $400 \%$ superior to the healthy tissue; this characteristic permits the ceMRI to accurately define the transmural extension of infarction and to distinguish among reversible and irreversible myocardium damage, independently from the contractile function of the wall, from the time of ischemic event and the state of perfusion. Furthermore, ceMRI has the incomparable advantage to permit a static and dynamic evaluation of the cardiac muscle in the three views of the space without exposing patient to ionizing radiations. Klein et al. [24], comparing the late enhancement in ceMRI and PET in patients with ischemic heart disease, showed that in patients with chronic ischemic left ventricular function, ceMRI is able to identify the location and the extension of the non-viable tissue with closely agreement with the results of the comparative study of blood flow and metabolism of glucose in PET. Nevertheless, in the evaluation of the infarct size, cardiac ceMRI seemed to underestimate the amount of necrotic tissue in comparison with nuclear technique [25]. This underestimation might be related to the low spatial resolution of nuclear imaging. Moreover, it was postulated that ceMRI may identify subendocardial infarcts, missed by nuclear technique. The quantitative analysis of ceMRI in patients with severe ischemic left ventricular dysfunction, demonstrated a larger extent of hyperenhancement in segments non-viable in PET imaging in comparison with viable segments [26]. Moreover, using a ROC analysis, a cut off value of $37 \%$ of 
hyperenhancement at ceMRI was identified to differentiate viable from non-viable segments. Finally, $\mathrm{Wu}$ et al. [27] analysed in 29 patients treated with surgical revascularization a similar value of sensitivity and specificity between ceMRI ad myocardial PET in predicting functional recovery. CeMRI predicted well the improvement of LVEF while in subjects with extensive necrotic tissue, myocardial PET was closely related to the poor early functional outcome.

This study compared the reliability of ceMRI and PET ${ }^{18} \mathrm{FDG}$ in the identification of the amount of myocardial tissue damage in ischemic left ventricular dysfunction. Our results confirmed that the presence of extension of delayed hyperenhancement in ceMRI is negatively correlated with the ${ }^{18}$ FDG uptake in PET. The semiquantitive analysis of the two imaging modalities gained a satisfactorily concordance between scores assigned in metabolic PET with ${ }^{18} \mathrm{FDG}$ and ceMRI. Finally we showed that, in the evaluation of transmural extension of contrast medium in ceMRI and uptake of ${ }^{18}$ FDG in PET study in graduated scale of 5 scores $(0-4)$, two experts observes obtained an agreement rate of $63 \%$ and $69 \%$. This experience demonstrated that in the visual evaluation of myocardial viability after myocardial infarction, ceMRI is accurate and effective in comparison with myocardial PET. This aspect offers important prospective in cardiological imaging, being MRI, compared to PET, largely diffused, more economical, faster and does not implicate radiological exposure.

\section{REFERENCES}

[1] Vogel-Claussen J, Fishman EK, Bluenke DA. Novel cardiovascular MRI and CT methods for evaluation of ischemic heart disease. Exp Rec Cardiovasc Ther 2007; 5(4): 791-802

[2] Pagano D, Lewis ME, Townend JN, et al. Coronarty revascularization for post-ischaemic heart failure : How myocardial viability affects survival. Heart 1999; 82: 684-8.

[3] Wu YW, Huang PJ, Lee CM, et al. Assessment of myocardial viability using F-18 fluorodeoxyglucose/Tc-99m sestamibi dualisotope simultaneous acquisition SPECT: comparison with Tl-201 stress-reinjection SPECT. J Nucl Cardiol 2005; 12: 451-9.

[4] Lin LC, Ho YL, Wu CC, et al. Comparison of simultaneous dobutamine echocardiography and thallium-201 stress-reinjection single-photon emission computed tomography in predicting improvement of chronic myocardial dysfunction after revascularization. Am J Cardiol 2000; 86: 293-8.

[5] Pagley PR, Beller GA, Watson DD, et al. Improved outcome after coronary bypass surgery in patients with ischemic cardiomyopathy and residual myocardial viability. Circulation 1997; 96: 793-800.

[6] Haas F, Haehnel CJ, Picker W, et al. Preoperative positron emission tomographic viability assessment and perioperative and postoperative risk in patients with advanced ischemic heart disease. J Am Coll Cardiol 1997; 30: 1693-700.

[7] Di Carli MF, Maddhai J, Rochsar S, et al. Long-term survival of patients with coronary artery disease and left ventricular dysfunction: implication of the role of myocardial viability assessment in management decisions. J Thorac Cardiovasc Surg 1998; 116: 9971004.

[8] Chaudhry FA, Tauke JT, Alessandini RS, et al. Prognostic implications of myocardial contractile reserve in patients with coronary artery disease and left ventricular dysfunction. J Am Coll Cardiol 1999; 34: 730-8.

[9] Baer FM, Voth E, Schneider CA, et al. Comparison of low dose dobutamine gradient echo magnetic resonance imaging and positron emission tomography with 18-FDG in patients with chronic coronary artery disease: a functional and morphological approach to the detection of the residual myocardial viability. Circulation 1995; 91 : 1006-15.

[10] Dilsizian V, Rocco TP, Freedman NMT, Leon MB, Bonow RO. Enhanced detection of ischemic but viable myocardium by the reinjection of thallium after stress-redistribution imaging. N Engl J Med 1990; 323: 141-6.

[11] Kuhl HP, Beek AM, van der Weerdt AP, et al. Myocardial viability in chronic ischemic heart disease: a comparison of contrast-enhanced magnetic resonance imaging with (18F)-fluorodeoxyglucose positron emission tomography. J Am Coll Cardiol 2003; 41: 1341-8.

[12] Maes A, Flameng W, Nuyts J, et al. Histological alterations in chronically hypoperfused myocardium: correlation with PET findings. Circulation 1994; 90: 735-45.

[13] Knuesel PR, Nanz D, Wyss C, et al. Characterization of dysfunctional myocardium by positron emission tomography and magnetic resonance: relation to functional outcome after revascularization. Circulation 2003; 108: 1095.

[14] Beek AM, Kuhl HP, Bondarenko O, et al. Delayed contrast-enhanced magnetic resonance imaging for the prediction of regional functional improvement after acute myocardial infarction. J Am Coll Cardiol 2003; 42: 895-901.

[15] Robert R. Edelman contrast-enhanced MR Imaging of the heart: overview of the literature. Radiology 2004; 232: 653-68.

[16] Judd RM, Lugo Olivieri CH, Arai AE, et al. Physiological basis of myocardial contrast enhancement in fast resonance images of 2 day old reperfused canine infarcts. Circulation 1995; 92: 1902-10.

[17] Kim RJ, Fieno DS, Parrish TB, et al. Relationship of MRI delayed contrast enhancement to irreversibile injury, infarct age and contractile function. Circulation 1999; 100: 1992-2002.

[18] Fieno DS, Kim RJ, Chen EL. Contrast enhanced magnetic resonance imaging of myocardium at risk: distinction between reversible and irreversible injury thought infarct healing. J Am Coll Cardiol 2000; 36: 1985-91.

[19] Hillenbrand HB, Kim RJ, Parker MA, et al. early assessment of myocardial salvage by contrast enhanced magnetic resonance imaging. Circulation 2000; 102: 1678-83.

[20] Cerqueira MD, Weissman NJ, Dilsizian V, et al. Standardised myocardial segmentation and nomenclature for tomographic imaging of the heart: a statement for healthcare professionals from the cardiac imaging committee of the Council of Clinical Cardiology of the American Heart Association. Circulation 2002; 105: 539-42.

[21] Karran LH. The reclutability of clinical methods, data and judments. N Engl J Med 1975; 6442-646.

[22] Pagano D, Lewis ME, Toned JN, et al. Coronary revascularization for post-ischaemic heart failure. How myocardial viability affects survival. Heart 1999, 82: 684-8.

[23] Pagley PR, Beller GA, Watson DD, et al. Improved outcome after coronary bypass surgery in patients with ischemic cardiomyopathy and residual myocardial viability. Circulation 1997; 96: 793-800.

[24] Klein C, Nekolla S, Bengel F, et al. Assessment of myocardial viability with contrast-enhancement magnetic resonance imaging. Comparison with positron emission tomography. Circulation 2002; 105: 162-7.

[25] Wu KC, Lima JAC. Non-invasive imaging of myocardial viability. Current techniques and future developments. Circ Res 2003; 93: 1146-58.

[26] Kuhl HP, Beek AM, van der Weerdt AP, et al. Myocardial viability in chronic ischemic heart disease. Comparison of contrast-enhanced magnetic resonance imaging with ${ }^{18} \mathrm{~F}$-Fluorodeoxyglucose positron emission tomography. J Am Coll Cardiol 2003; 41: 1341-8.

[27] Yen WW, Ejii T, Masaki Y, et al. Comparison of contrast-enhanced MRI with 18-F-FDG PET/ $201 \mathrm{Tl}$ SPECT in dysfunctional myocardium: relation to early functional outcome after surgical revascularization in chronic ischemic heart disease. J Nucl Med 2007; 48(7): 1096-103. 